\section{Randomized Controlled Trial of a Psychoeducational Video Intervention for Traumatic Injury Survivors}

\section{Eunice C Wong $^{1 *}$, Grant N Marshall ${ }^{1}$ and Jeremy NV Miles ${ }^{1}$}

\begin{abstract}
Objective: This randomized controlled trial evaluated the impact of a psychoeducational video on posttraumatic distress and factors related to the mental health treatment seeking process among trauma care center patients receiving care following hospitalization for a serious physical injury.
\end{abstract}

Method: Ninety-nine, predominantly Hispanic American (59\%) and male $(84 \%)$, participants were randomized to view either a psychoeducation intervention or a wound care control video. Participants were assessed at pre-intervention, post-intervention, and at 1-month follow-up on posttraumatic stress reactions, attitudes toward mental health service utilization, and knowledge and self-recognition of posttraumatic stress symptoms.

Results: Immediately after viewing the psychoeducational video, participants exhibited greater knowledge of PTSD symptoms and more positive beliefs about mental health treatment than those in the wound care condition. At 1-month follow-up, however, these differences were no longer maintained. Further, no significant differences in PTSD were found between the intervention and control groups. Differences in self-recognition of PTSD nearly reached significance with psychoeducation intervention participants being more likely to recognize their symptoms as mental health problems than control condition participants.

Conclusion: Brief psychoeducation interventions for traumatic injury survivors may have had immediate modest effects on mental health literacy, beliefs about mental health treatment, and selfrecognition of PTSD, but were not maintained at 1-month follow-up. More comprehensive and targeted interventions may be needed to facilitate more potent and long-lasting change.

\section{Keywords}

Psychoeducation; Posttraumatic stress disorder; Trauma; Brief intervention

\section{Introduction}

Approximately two million Americans are hospitalized for a physical injury each year [1]. A substantial proportion, anywhere from $20 \%$ to $30 \%$, goes on to develop posttraumatic stress disorder (PTSD) [2,3]. Increasingly, psychoeducation is being routinely

\footnotetext{
* ${ }^{*}$ orresponding author: Eunice C Wong, RAND Corporation, 1776 Main St. P.O. Box 2138, Santa Monica, CA, 90407-2138, USA, Tel: 310-393-0411 ext. 6389; Fax: 310-260-8153; E-mail: ewong@rand.org
}

Received: January 30, 2012 Accepted: March 29, 2013 Published: April 05, 2013 delivered during the aftermath of a trauma [4-7]. Unlike other mental disorders, posttraumatic stress reactions can be linked to a specific point in time (i.e., traumatic event). This timing may be advantageous for providing psychoeducation immediately after a trauma as a cost-efficient prevention intervention [8]. Psychoeducation often includes providing information about common posttraumatic stress reactions and sources of assistance [4]. The underlying premise is that psychoeducation can help trauma survivors to better understand and normalize common posttraumatic stress reactions, and improve their recognition of when mental health treatment is indicated and how to obtain it $[9,10]$.

Despite the increasingly widespread practice of providing psychoeducation during the aftermath of a trauma, little effort has been devoted to the empirical study of its effectiveness $[4,8,11]$. Wessely et al. [4] found only a single study that directly tested the effects of providing psychoeducation following trauma. This study involved a randomized controlled trial (RCT) in which trauma care patients who were given psychoeducational self-help booklets fared no better than those who did not in terms of posttraumatic stress symptoms [12]. In fact, among patients with PTSD, the control group appeared to improve the most, suggesting that the provision of psychoeducation might have been detrimental for the most distressed individuals. Other studies have found minor benefits for psychoeducation when compared as a control group to PTSD psychotherapy interventions (e.g., cognitive behavioral therapy [CBT], narrative exposure therapy) [4]. However, in one RCT, trauma survivors who had received psychoeducation exhibited worse outcomes compared to not only those in the CBT condition, but also to those in the assessment only condition [13].

Given the state of empirical knowledge on the value of psychoeducation as a prevention intervention, the posited benefits of providing psychoeducation following trauma have come under serious scrutiny with some even calling into question whether such a practice might be harmful $[4,8]$. To determine whether routinely offering psychoeducation after a traumatic event is warranted, further research assessing the impact of psychoeducation on the prevention of posttraumatic distress and promotion of recovery is needed $[8,11]$. Such research could include evaluating the effect of psychoeducation on factors that facilitate the help seeking process as well as posttraumatic distress.

This study examined the effects of a psychoeducational video intended for trauma care center patients recovering from a serious physical injury. The psychoeducational video featured actual traumatic injury survivors who provided information on PTSD symptoms, overcoming barriers to treatment, and the process and benefits of obtaining mental health treatment. Although psychoeducation can also encompass the provision of strategies to more effectively cope with PTSD symptoms (e.g., information about exposure), the present study focused solely on providing information about common posttraumatic stress reactions and the help-seeking process, given critiques that such information may exacerbate symptoms or symptom reporting. Participants were randomly assigned to view videos presenting information either on PTSD psychoeducational material or information on self-care for physical 
wounds. The following outcomes were assessed: PTSD symptoms, knowledge and self-recognition of PTSD, and beliefs about mental health treatment. As an exploratory aim, we examined the impact of the psychoeducational video on mental health treatment utilization given that no study has tested whether psychoeducation does in fact aid in the help seeking process [4].

\section{Methods}

\section{Participants}

Participants were recruited from a large trauma care center in Los Angeles County. Research staff had direct access to computerized admission records, which were used to identify all admitted patients over 18 years of age whose injuries required surgical intervention for injuries consistent with trauma registry criteria (e.g., gunshot wound, falls from greater than 15 feet). Patients who were non-English speaking, in criminal custody, or unable to provide informed consent because of severe cognitive impairment were excluded. Interviewers attempted to screen and consent all consecutively-admitted eligible patients. Of the 101 patients who were approached for the study, $99(98 \%)$ consented to participate and were randomized to the psychoeducation or wound care control condition. Of these, $79(80 \%)$ completed the 1-month follow-up interview (Figure 1).

\section{Procedures}

All study procedures were approved by the Institutional Review Boards of the RAND Corporation and the Los Angeles County/ University of Southern California Medical Center. After providing informed consent, participants were randomly assigned to view either the psychoeducation intervention (18 minutes) or wound care control video (10 minutes). A block randomization procedure was used to ensure equal distributions of gender and injury mechanism (i.e., assault vs. motor vehicle accident) across the two conditions. Participants completed a baseline assessment interview prior to viewing the assigned video on a portable DVD player. Immediately after viewing the video, participants completed a post-video interview

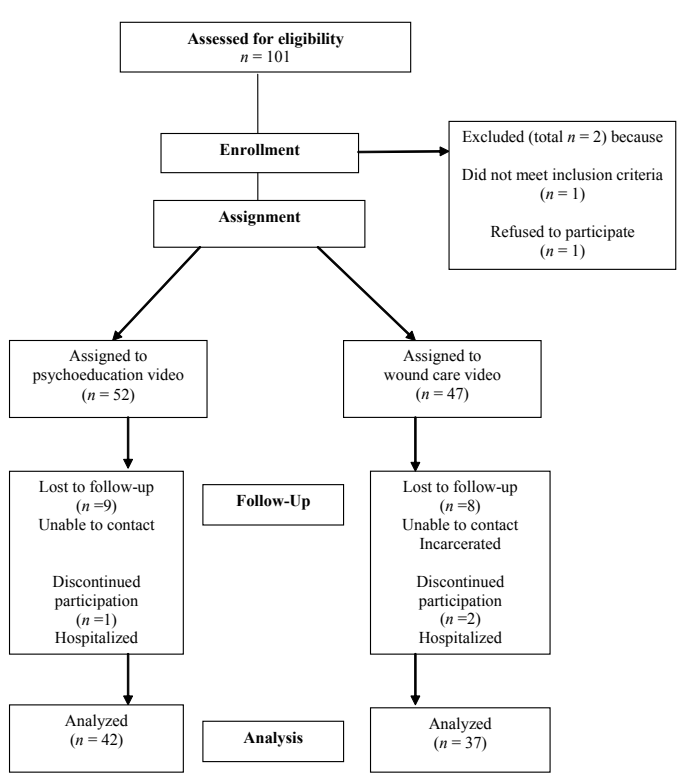

Figure 1: Participant flow from baseline interview to one-month follow-up. assessment. The 1-month follow-up interviews were conducted by phone. Participants were paid $\$ 30$ each time after the post-video and the 1-month follow-up assessment.

\section{Measures}

The baseline interview obtained information on demographics (e.g., age, ethnicity), injury type, PTSD symptoms, mental health literacy, mental health treatment beliefs and use. The post-video interview reassessed mental health literacy and treatment beliefs. The 1-month follow-up interview assessed PTSD symptoms, mental health literacy and beliefs, self-recognition of mental health problems, and treatment use.

Posttraumatic stress reactions: The Posttraumatic Stress Disorder Checklist (PCL) was used to assess for probable PTSD and symptom severity [14]. The PCL requires participants to rate the degree to which they were bothered by each of 17 PTSD symptoms on a scale from 1 (not at all) to 5 (extremely). For probable PTSD, the PCL was scored according to the Diagnostic Statistical Manual of Mental Disorders Symptoms, $4^{\text {th }}$ Edition [15], by requiring endorsement of one re-experiencing, three avoidance/numbing, and two hyperarousal symptoms at a clinically significant level. A score of 3 or more was considered a clinically significant [14]. PTSD symptom severity was derived by using the total sum score. The PCL demonstrated good internal consistency at baseline $(\alpha=0.88)$ and follow-up $(\alpha=0.89)$. The PCL was used as an approximate measure for posttraumatic stress reactions immediately following a traumatic physical injury, even though the PCL is commonly used as a measure for PTSD that is administered at least one month after trauma exposure. The PCL has been used in prior research to assess immediate posttraumatic stress reactions among hospitalized trauma care center patients [16,17] and has been found to the single best predictor of subsequent PTSD symptoms as measured by the PCL at follow-up [18].

Knowledge and self-recognition of PTSD: The Knowledge of PTSD Test [19] was modified to assess knowledge about traumatic events, posttraumatic stress reactions, and treatment. The measure consisted of 8 multiple-choice items and a total score was derived by summing the number of correct responses. To assess self-recognition of mental health problems, a single (yes/no) item adapted from the National Latino and Asian American Study was used [20], which asked participants if there was any time when they thought they had problems with posttraumatic stress disorder.

Mental health treatment beliefs: The Beliefs about Psychotherapy (BAP)and Beliefs about Psychotropic Medication (BAPM) scales [21] assessed beliefs about psychotherapy and psychotropic medication. All items were rated on a Likert scale from 1 (strongly disagree) to 5 (strongly agree) with greater mean scores reflecting more positive beliefs. The BAP and BAPM demonstrated adequate reliability at baseline ( $\alpha=0.84$ and $\alpha=0.73$, respectively) and follow-up ( $\alpha=0.68$ and $\alpha=0.70$, respectively).

Mental health service use was assessed by a single (yes/no) item that asked, "Since your last interview, have you seen a health professional (e.g., psychiatrist, psychologist, doctor, and counselor) for personal or emotional problems such as with your mood, nerves, alcohol, drugs, or mental health?"

\section{Treatment conditions}

Psychoeducation: The content of the psychoeducational video (18 minutes) was based upon the commonsense model of self- 
regulation [22]. According to the commonsense model, perceptions or beliefs about symptoms can vary along five key dimensions (i.e., identity, causes, consequences, controllability, and timeline) that influence how individuals cope with an illness including whether to seek treatment. The psychoeducational video targeted these key dimensions by providing information about traumatic events and common posttraumatic stress reactions (causes/identity), the potential impact of PTSD on functioning (consequences), the benefits of obtaining treatment (controllability), and when treatment might be indicated (timeline). Information was delivered through a host narrator and four traumatic injury survivors who shared information about their posttraumatic stress experiences as well as the process and benefits of obtaining treatment.

Wound care: The wound care video (10 minutes), "Lacerations and Wound Care," was produced by an organization that develops patient health education videos for use by hospital and clinics [23]. Topics covered included medical treatment for lacerations, the healing process, and home care.

\section{Analyses}

Analyses controlled for PTSD symptoms for outcome variables that have been shown to be or are likely to be associated with psychological distress (i.e., beliefs about treatment, self-recognition of PTSD, and mental health service use). Linear regression analyses were used to compare the impact of the psychoeducational and the wound care videos on continuous outcome variables (e.g., beliefs about psychotherapy). Logistic regression analyses were used to examine the impact of the interventions on categorical outcome variables (e.g., probable PTSD).

\section{Results}

\section{Participant characteristics}

Participants were predominantly male (84\%) and largely selfidentified as Hispanic American (59\%). Their mean age was 31 years old and approximately $32 \%$ had experienced an assault-related traumatic injury (e.g., shot, stabbed, kicked, or hit). The mean Injury Severity Score (ISS) was 4.99 (SD=1.35) [24]. Scores of 9 and below are considered to be of mild to moderate severity; however, all injuries in the present study were serious enough to require hospitalization. At baseline, among participants in the psychoeducation condition, $13.5 \%$ met probable criteria. No significant differences were found between the psychoeducation and control group at baseline (Table 1).

Immediately after viewing the videos, participants in the psychoeducation condition exhibited greater PTSD knowledge $(\mathrm{p}<0.05)$ and held more positive views toward psychotherapy $(\mathrm{p}<0.001)$ and medication $(\mathrm{p}<0.001)$ than those in the wound care condition. One month after viewing the videos, follow-up phone interviews were conducted to assess for PTSD symptoms as well as for the maintenance of any gains in mental health literacy or beliefs about mental health treatment. No significant differences in PTSD were found between the psychoeducation and wound care conditions. Specifically, the psychoeducation and wound care conditions did not differ with respect to rates of probable PTSD ( $\mathrm{p}=0.83$ ) or PTSD symptom severity $(\mathrm{p}=0.42)$. Further, the immediate post-intervention gains found for the psychoeducation condition were no longer maintained at one-month follow-up. Differences in PTSD knowledge and mental health treatment beliefs were no longer found between the psychoeducation and wound care conditions (Table 2).
At one-month follow-up, participants were also assessed on selfrecognition of mental health problems and on mental health service use. Approximately $14 \%$ of participants reported seeing a health professional for mental health problems at follow-up. No significant differences in treatment use were found between the two conditions (Table 2). However, group differences in self-recognition of PTSD narrowly failed to achieve significance. Controlling for PTSD symptoms, participants in the psychoeducation condition were more likely to endorse self-recognition of PTSD problems than those in the control condition $[\mathrm{OR}=4.27,95 \% \mathrm{CIs}=1.00,18.43, \mathrm{p}=0.05]$.

\section{Discussion}

This study responds to the numerous calls for randomized controlled investigations that examine whether psychoeducation, as a stand-alone intervention, supports or hinders the trauma recovery process $[8,11,25]$. To the authors' knowledge, this is also the first study to examine the putative effects of psychoeducation on the help seeking process. Amidst growing concerns about the potentially harmful effects of psychoeducation $[4,8]$, this study found no evidence that psychoeducation adversely affected traumatic injury survivors, in so far as, no differences in PTSD were found between psychoeducation and control condition participants at 1-month follow-up. This finding contrasts with other studies suggesting that trauma injury survivors may actually fare worse when given psychoeducation via written materials $[12,13]$.

Although our findings provide no corroborating evidence for the injurious effects of psychoeducation, neither do they provide supporting evidence for the preventative effects of psychoeducation on posttraumatic stress reactions. A common, yet rarely tested, assumption is that psychoeducation may mitigate posttraumatic stress reactions; for example, by normalizing experiences or facilitating access to treatment during the aftermath of a trauma [4,9]. Our findings did not support this assumption in that reductions in PTSD symptoms were not evidenced among those in the psychoeducation condition relative to those in the wound care condition.

With respect to the impact of psychoeducation on the helpseeking process, our study found short-term effects on mental health treatment beliefs and mental health literacy. Immediately after viewing the videos, participants who were exposed to the psychoeducational video demonstrated greater knowledge of posttraumatic stress reactions and more positive beliefs about psychotherapy and psychotropic medications than those who viewed the wound care

Table 1: Demographic and baseline information.

\begin{tabular}{|l|c|c|c|c|c|c|c|}
\hline & \multicolumn{3}{|c|}{ Psychoeducation } & \multicolumn{3}{c|}{ Wound care } \\
\hline Variables & \multicolumn{2}{|c|}{$(\mathrm{n}=52)$} & & \multicolumn{3}{c|}{$(\mathrm{n}=47)$} & \\
\hline Age (years) & 28.8 & & 9.05 & 33.7 & & 12.3 & 0.03 \\
\hline Years in school & 12.4 & & 1.7 & 12.1 & & 2 & 0.45 \\
\hline & & $\%$ & & & $\%$ & & \\
\hline Female & & 17.3 & & & 14.9 & & 0.79 \\
\hline Ethnicity & & & & & & & 0.28 \\
\hline African American & & 13.5 & & & 10.6 & & \\
\hline Asian American & & 15.4 & & & 4.3 & & \\
\hline Hispanic & & 55.8 & & & 61.7 & & \\
\hline White & & 11.5 & & & 21.3 & & \\
\hline Other & & 3.9 & & & 2.1 & & \\
\hline Assault Type Injury & & 32.7 & & & 31.9 & & 1 \\
\hline Probable PTSD & & 13.5 & & & 8.5 & & 0.53 \\
\hline & & & & & & & \\
\hline
\end{tabular}


Table 2: Linear and Logistic Regression Analyses of PTSD knowledge, Beliefs about Mental Health Treatment, PTSD, Self-Recognition and Mental Health Service Utilization as a Function of the Intervention Group.

\begin{tabular}{|c|c|c|c|c|c|c|}
\hline \multirow[b]{3}{*}{ Outcome Variable } & \multicolumn{3}{|c|}{ Immediate } & \multicolumn{3}{|c|}{ One-Month } \\
\hline & \multicolumn{3}{|c|}{ Post-Intervention } & \multicolumn{3}{|c|}{ Post-Intervention } \\
\hline & $\beta$ & $\mathrm{P}$ & $95 \% \mathrm{Cls}$ & $\beta$ & $\mathrm{P}$ & $95 \% \mathrm{Cls}$ \\
\hline PTSD knowledge & 0.57 & 0.028 & $0.06,1.07$ & 0.24 & 0.631 & $0.06,1.07$ \\
\hline Beliefs about psychotherapy & 0.61 & $<.001$ & $0.49,0.74$ & 0.03 & 0.735 & $-0.17,0.23$ \\
\hline \multirow[t]{2}{*}{ Beliefs about psychotropic medication } & 0.57 & $<.001$ & $0.43,0.72$ & 0.06 & 0.648 & $-0.21,0.34$ \\
\hline & OR & $\mathrm{P}$ & $95 \% \mathrm{Cl}$ & OR & $\mathrm{P}$ & $95 \% \mathrm{Cl}$ \\
\hline Probable PTSD & - & & - & 0.79 & 0.764 & $0.23,2.67$ \\
\hline Self-recognition of PTSD symptoms & - & & - & 4.27 & 0.052 & $0.99,18.43$ \\
\hline Mental health service use & - & & - & 1.02 & 0.843 & $0.25,4.25$ \\
\hline
\end{tabular}

Note: For linear regression analyses, positive values indicate higher scores for the psychoeducational video than the wound care video. For logistic regression analyses, the wound care condition was coded 0 and the psychoeducation condition was coded 1 . OR = odds ratio; $\mathrm{Cl}=$ confidence interval

video. Nevertheless, these group differences in mental health literacy and beliefs were no longer maintained at one-month follow-up. Our findings suggest that psychoeducation can impact mental health literacy and beliefs upon immediate exposure. Yet, these effects do not appear to be long lasting. Findings highlight the importance of examining the process of psychoeducation, which includes not only changes in knowledge and beliefs but also the degree of uptake and comprehension of information [8].

The psychoeducational video, however, did yield potentially promising, longer term effects on participants' self-recognition of PTSD symptoms. At one-month follow-up, controlling for PTSD symptoms, participants who viewed the psychoeducational video were more likely to recognize themselves as having problems with PTSD compared to those who had viewed the wound care video. These group differences narrowly failed to achieve significance at the conventional $\mathrm{p}=0.05$ level. Not recognizing PTSD symptoms as problematic has been implicated as a significant treatment barrier. For example, in a nationally representative U.S. sample, $66 \%$ of individuals who met criteria for PTSD reported that they had not sought help because they did not perceive themselves as having a problem [26].

Our exploratory analyses revealed that viewing the psychoeducational video did not result in increased service utilization. This is not unexpected given that the study was most likely insufficiently powered to detect such effects. Also, major practical barriers (e.g., treatment costs, transportation difficulties), which have been documented among individuals with PTSD, may have impeded service utilization [26]. Further study is needed to assess whether psychoeducation that is targeted at an array of attitudinal and structural barriers can successfully facilitate the obtainment of needed treatment. For instance, little investigation has focused on whether improving mental health literacy and beliefs about mental health treatment would result in increased utilization of services.

Findings must be considered in light of certain study limitations This study examined the effects of a relatively brief psychoeducational intervention. Further research examining the effects of stronger doses or booster sessions of psychoeducation on maintaining and improving changes in mental health literacy, beliefs, and service use may be warranted. Moreover, the psychoeducation intervention in this study focused on perceptions or beliefs that have been implicated in the symptom appraisal and help-seeking process [22]. Other types of psychoeducation such as providing information on adaptive coping strategies that foster resiliency may also be promising avenues to pursue $[4,11,27]$. The study may have also been insufficiently powered to detect intervention effects on self-recognition of PTSD symptoms and treatment seeking given that these outcomes may have been mostly relevant for the proportion that met criteria for probable PTSD. Nevertheless, this study's aim was to examine the impact of a psychoeducational video on the recovery process for traumatic injury survivors and findings are limited to this population. Finally, the study's findings may have been influenced by gender or cultural factors given that participants were mostly male and Hispanic American, which may limit the generalizability of the findings.

In conclusion, although this study yielded only weak evidence of the impact of psychoeducation on mental health beliefs and PTSD knowledge, it did not appear to have detrimental effects at least in the short-term. Psychoeducation may be able to achieve its promise, but only with additional attention to understanding how to better target and deliver such interventions so that maximum benefits can be reaped.

\section{Acknowlegement}

This research was supported by grants R34MH071569, R21DE014973, $\mathrm{R} 01 \mathrm{MH} 056122$, and R01AA014246. We are also indebted to the research participants without whom this study would not have been possible.

\section{References}

1. Bonnie RJ, Fulco CE, Liverman CT (1999) Reducing the burden of injury: Advancing prevention and treatment. Washington, DC: National Academy Press.

2. O'Donnell ML, Creamer M, Pattison P (2004) Posttraumatic stress disorder and depression following trauma: understanding comorbidity. Am J Psychiatry 161: 1390-1396.

3. Shih RA, Schell TL, Hambarsoomian K, Belzberg H, Marshall GN (2010) Prevalence of posttraumatic stress disorder and major depression after trauma center hospitalization. J Trauma 69: 1560-1566.

4. Wessely S, Bryant RA, Greenberg N, Earnshaw M, Sharpley J, et al. (2008) Does psychoeducation help prevent post traumatic psychological distress? Psychiatry 71: 287-302.

5. National Institute for Health and Clinical Excellence (2005) Post-Traumatic Stress Disorder: The Management of PTSD in Adults and Children in Primary and Secondary Care. National Collaborating Centre for Mental Health (UK).

6. World Health Organization (2003) Mental Health in Emergencies. Mental and Social Aspects of Health of Populations Exposed to Extreme Stressors. World Health Organization Geneva.

7. National Institute of Mental Health (2002) Mental Health and Mass Violence: Evidence-Based Early Psychological Intervention for Victims/Survivors of Mass Violence. A Workshop to Reach Consensus on Best Practices. Washington, D.C: U.S.

8. Creamer M, O'Donnell M (2008) The pros and cons of psychoeducation following-trauma: too early to judge? Psychiatry $71:$ 319-321. 
Citation: Wong EC, Marshall GN, Miles JNV (2013) Randomized Controlled Trial of a Psychoeducational Video Intervention for Traumatic Injury Survivors. J Trauma Stress Disor Treat 2:2.

9. Phoenix BJ (2007) Psychoeducation for survivors of trauma. Perspect Psychiatr Care 43: 123-131.

10. Southwick S, Friedman M, Krystal J (2008) Does psychoeducation help prevent post traumatic psychological stress disorder? In reply. Psychiatry 71 303-307.

11. Krupnick JL, Green BL (2008) Psychoeducation to prevent PTSD: a paucity of evidence. Psychiatry 71: 329-331.

12. Turpin G, Downs M, Mason S (2005) Effectiveness of providing self-help information following acute traumatic injury: randomised controlled trial. $\mathrm{Br}$ Psychiatry 187: 76-82.

13. Ehlers A, Clark DM, Hackmann A, McManus F, Fennell M, et al. (2003) A randomized controlled trial of cognitive therapy, a self-help booklet, and repeated assessments as early interventions for posttraumatic stress disorder. Arch Gen Psychiatry 60: 1024-1032.

14. Weathers FW, Litz BT, Herman DS, Huska JA, Keane TM (1993) The PTSD Checklist (PCL): Reliability, validity, and diagnostic utility. In: meeting of the International Society for Traumatic Stress Studies. San Antonio, TX, 24-27.

15. American Psychiatric Association (1994) Diagnostic and statistical manual of mental disorders. 4th edn. Washington, DC.

16. Wong EC, Schell TL, Marshall GN, Jaycox LH, Hambarsoomians K, et al. (2009) Mental health service utilization after physical trauma: the importance of physician referral. Med Care 47: 1077-1083.

17. Zatzick D, Roy-Byrne P, Russo J, Rivara F, Droesch R, et al. (2004) A randomized effectiveness trial of stepped collaborative care for acutely injured trauma survivors. Arch Gen Psychiatry 61: 498-506.

18. Denson TF, Marshall GN, Schell TL, Jaycox LH (2007) Predictors of posttraumatic distress 1 year after exposure to community violence: the importance of acute symptom severity. J Consult Clin Psychol 75: 683-692.

19. Pratt SI, Rosenberg S, Mueser KT, Brancato J, Salyers M, et al. (2005) Evaluation of a PTSD psychoeducational program for psychiatric inpatients. Journal of Mental Health 14: 121-127.

20. Alegria M, Takeuchi D, Canino G, Duan N, Shrout P, et al. (2004) Considering context, place and culture: the National Latino and Asian American Study. Int J Methods Psychiatr Res 13: 208-220.

21. Bystritsky A, Wagner AW, Russo JE, Stein MB, Sherbourne CD, et al. (2005) Assessment of beliefs about psychotropic medication and psychotherapy: development of a measure for patients with anxiety disorders. Gen Hosp Psychiatry 27: 313-318.

22. Leventhal $H$, Leventhal EA, Cameron $L$ (2001) Representations, procedures and affect in illness self-regulation: A perceptual-cognitive model. Handbook of health psychology. Mahwah, New Jersey: Lawrence Erlbaum Associates, $19-47$.

23. Association for the Advancement of Automotive Medicine (1990) The Abbreviated Injury Scale (revision). Des Plaines, IL.

24. www.wired.md

25. Ruzek JI (2008) Wanted: a theory of post-trauma information delivery Psychiatry $71:$ 332-338.

26. Kessler RC (2000) Posttraumatic stress disorder: the burden to the individual and to society. J Clin Psychiatry 61: 4-12.

27. Resnick H, Acierno R, Holmes M, Kilpatrick DG, Jager N (1999) Prevention of post-rape psychopathology: preliminary findings of a controlled acute rape treatment study. J Anxiety Disord 13: 359-370.
Author Affiliation

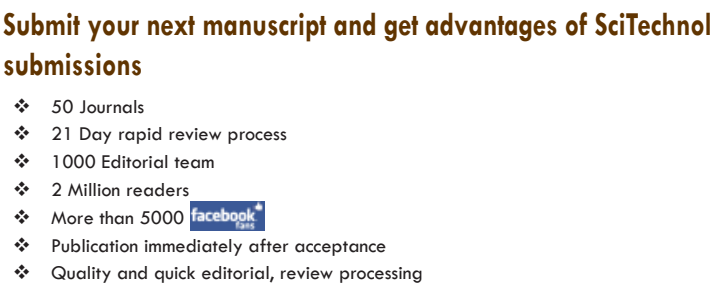

Submit your next manuscript at • www.scitechnol.com/submission 